\title{
Strategies to Combat Hypoxia in Encapsulated Islet Transplantation
}

Rahul Krishnan ${ }^{1}$, David Ko ${ }^{1}$, Tori Tucker ${ }^{1}$, Emmanuel Opara ${ }^{2}$, Clarence E Foster III', David Imagawa ${ }^{1}$, Michael Stamos ${ }^{1}$ and Jonathan RT Lakey ${ }^{1,3^{*}}$

${ }^{1}$ Department of Orthopedic Surgery, Southampton University Hospitals NHS Trust, Pakistan

${ }^{2}$ Department of Orthopedic Surgery, Klinikum Neumarkt, Germany

${ }^{3}$ Department of Biomedical Engineering, University of California, USA

\begin{abstract}
Islet transplantation has been shown as a possible treatment for Type 1 Diabetes. However, immediately following transplantation, islets face acute hypoxic stress due to the lack of vascularization of the newly transplanted tissue. It has been observed that up to $60 \%$ of newly transplanted islets perish during the first 48 hours post-transplantation as a direct result of hypoxic injury. This period of hypoxia needs to be reduced to maintain transplanted islet efficacy. Optimal function of immunoisolated islets requires adequate supply of oxygen to metabolically active insulin producing $\beta$-cells. An improved understanding of the interplay between oxygen diffusion and consumption rate in devices is critical for the design of means to improve oxygen supply to islets. Post-transplant graft failure and islet death have been postulated to result from hypoxia encountered immediately after transplantation, due to poor implant-site vascularization. In order to survive and function adequately, transplanted islets must be able to withstand the transient hypoxic shock until they are adequately vascularized. This review will explore the various mechanisms that result in loss of cell viability and insulin release from islets when they are exposed to hypoxic conditions, and summarize the various strategies that are being employed by researchers across the world to address this important issue to enable rapid translation of results obtained in pre-clinical trials to primate and human trials.
\end{abstract}

Keywords: Oxygen; Diffusion; Microcapsule; Encapsulation; Islet; OCR; Necrosis; Hypoxia; Angiogenesis

\begin{abstract}
Abbreviations: T1D: Type 1 Diabetes; PCO: Pericapsular Overgrowth; OCR: Oxygen Consumption Rate; HBO: Hyperbaric Oxygen Therapy; EX-4: Exendin-4; FGF-1: Fibroblast Growth Factor 1; aFGF: acidic Fibroblast Growth Factor; VEGF: Vascular Endothelial Growth Factor;VEGFR2: Vascular Endothelial Growth Factor Receptor 2; AMPK: Adenosine Monophosphate Activated Protein Kinase ; NFkB: Nuclear Factor $\kappa$-light chain enhancer of activated B-Cells; PDMS: Polydimethylsiloxane; n PTFE: Polytetrafluoroethylene; HIF1a: Hypoxia-InducibleFactor-1a; HIMP1: Hypoglycemia/hypoxia Inducible Mitochondrial Protein; BNIP-3: BCL2/adenovirus E1B 19 $\mathrm{kDa}$ protein-interacting protein 3; Bcl-2: B-cell lymphoma 2; PDX-1: Pancreatic and duodenal homeobox 1;HO-1: Heme Oxygenase-1; HSP32: Heat Shock Protein-32; APA: Alginate-Poly-L-Lysine-Alginate; PLO: Poly-L-Ornithine; PFC: Perfluorocarbon; BAP: Bioartificial Pancreas; NPI: Neonatal Porcine Islets; LVG: Low Viscosity High Guluronate; TUNEL: Terminal deoxynucleotidyl transferase dUTP nick end labeling; UPMVG: UltraPure Medium Viscosity High Guluronate; ATP: Adenosine Triphosphate; ADP: Adenosine Diphosphate; GHRH:Growth Hormone Releasing Hormone; CYGB: Cytoglobin; COX-2: Cyclooxygenase - 2; Hb-C: Crosslinked Hemoglobin; IEQ: Islet Equivalents; $\mathrm{NCX} 1: \mathrm{Na}^{+}-\mathrm{Ca}^{++}$Exchanger 1; $\mathrm{MT} 1 / 2$ : Metallothionein 1/2; MT-II: Metallothionein - II; MCP-1: Macrophage Chemoattractant Protein -1; XIAP: X-linked Inhibitor of Apoptosis Protein; MIN6: Mouse Insulinoma 6; GSIR: Glucose Stimulated Insulin Response; $\mathrm{CaO}_{2}$ : Calcium Peroxide; $\mathrm{SrCl}_{2}$ : Strontium Chloride; MSC: Mesenchymal Stem Cell
\end{abstract}

\section{Introduction}

Diabetes is a widespread global health problem, affecting approximately 382 million people worldwide in 2013, and 22 million in United States [1]. The figures are trending upwards, with the number of diabetics projected to reach 592 million by 2035 [2]. The conventional treatment paradigm of lifelong exogenous insulin administration for the management type 1 diabetes (T1D) is not without its disadvantages. The exacting demands of frequent injections and blood glucose monitoring leads to poor patient compliance, leading to suboptimal blood glucose homeostasis. In the long-term, this leads to several microvascular complications and severe end-organ damage, such as cardiovascular disease, neuropathy, retinopathy and nephropathy. Pancreatic islet transplantation is a promising alternative that affords prolonged endogenous regulation of glycemic control, thus supplanting the need for exogenous insulin therapy. However, only a handful of institutions worldwide have been able to demonstrate consistent success, thus restricting its availability to type 1 diabetics with severe end organ damage. A crippling scarcity of cadaver pancreas donors and the need for multiple donors to ensure an adequate dose of islets is available for transplantation also restrict its wider application.

To circumvent this problem in supply, researchers have attempted to utilize xenogeneic islets of porcine origin. However, transplant studies in mouse, rat, and non-human primate models have only reported transient improvements in glycemic indices despite the use of immunosuppressive drugs. In the rare cases that the grafts survived acute or chronic immune-mediated destruction, the adverse effects associated with the immunosuppressive therapy required to preserve the grafts, precluded clinical application of islet xenotransplantation. When faced with this obstacle, investigators proposed islet encapsulation, creating an immunoisolation barrier around these xenografts to prevent them from activating the immune system and protect them from cytotoxic sentinel cells of the recipient's immune system.

*Corresponding author: Jonathan RT Lakey, Associate Professor, Department of Surgery \& Biomedical Engineering, Director of Surgical Research, Director of Clinical Islet Program, University of California, Irvine,333 City Blvd W Suite 1600, Orange, California, 92868, USA, Tel: +1 (714) 851 8856; E-mail: jlakey@uci.edu

Received December 28, 2015; Accepted February 03, 2016; Published February 11,2016

Citation: Krishnan R, Ko D, Tucker T, Opara E, Foster CE, et al. (2016) Strategies to Combat Hypoxia in Encapsulated Islet Transplantation. Surgery Curr Res 6: 259 doi:10.4172/2161-1076.1000259

Copyright: @ 2016 Krishnan R, et al. This is an open-access article distributed under the terms of the Creative Commons Attribution License, which permits unrestricted use, distribution, and reproduction in any medium, provided the original author and source are credited. 
Although encapsulated islet transplants succeeded in achieving prolonged xenogeneic graft functionality in several studies, a primary factor hindering long-term functionality of the islets was a significant reduction in tissue oxygenation. Hypoxia is detrimental to $\beta$ cell function. Hypoxia upregulates pro-apoptotic genes such as HIF-1 $\alpha$, caspase-3, and BNIP-3 [3], stimulates increased generation of reactive oxygen species, especially nitric oxide (NO) [4] and triggers the downregulation of anti-apoptotic genes, such as Bcl-2, and PDX-1 [5], all of which result in decreased cell viability. Furthermore, hypoxia also precipitates amplification of gene products that have immunecell stimulating affects, such as MCP-1 [6]. These ultimately severely compromise the ability of transplanted islet grafts to regulate glucose homeostasis.

Islets are metabolically highly active and inherently consume a disproportionate amount of oxygen compared to neighboring pancreatic exocrine acinar tissue. Often, they utilize $5-10 \%$ of pancreatic blood flow for adequate perfusion while only constituting $1-2 \%$ of pancreatic mass [7]. Additionally, the isolation process disrupts the vascular networks that are required to deliver sufficient oxygen, as the islets are removed from their native microenvironment. The process of re-establishing microvascular connections with the recipient takes several days to a few weeks; often mature capillaries fail to form, or are prone to early thrombosis or collapse, thus resulting in graft failure and necrosis. In encapsulated islets, the immunoisolation layer acts as a physical barrier, preventing the formation of the islet-recipient microvascular nexus. This renders the islets extremely vulnerable to hypoxic injury as the islets now depend solely on passive diffusion of oxygen from the microenvironment for their survival and function. The establishment of a physical boundary significantly increases the diffusion distance, which decreases oxygen permeability. Thus, if the geometry and composition of the encapsulating material is chosen, the diffusion barriers can be minimized to improve oxygen delivery.

Encapsulated islets cannot be safely transplanted into the liver through the portal vein, the conventional site of choice in islet transplantation, as the significantly greater graft volume, the geometry and the material of the encapsulant would result in either portal vein occlusion or thrombosis. This has necessitated research evaluating alternative transplant sites such as the omentum, the peritoneal space, or the subcutaneous space. None of these sites are as vascular as the liver, or the native pancreas. Finally, the formation of pericapsular fibrotic overgrowth (PCO), whereby the adhesion of host cells on the surface of the capsule further impedes diffusive transfer, and worsens hypoxic conditions experienced by islets.

The following review highlights several possible strategies to ameliorate hypoxia in encapsulated islet grafts. The importance of identifying appropriate donor parameters, tissue conditions, biomaterial device geometry and composition, and recipient transplant sites, and preconditioning or co-encapsulating donor islets with bioactive compounds or oxygen, to mitigate hypoxic injury to achieve better transplant outcomes, will be discussed in detail with the goal of translating this success to clinical trials in human type 1 diabetic patients

\section{Mechanisms of hypoxic injury in unencapsulated islets}

Hypoxia is a crucial factor in cell adaptation and environmental stress. The ischemic and mechanical injury caused by islet isolation causes increased post-transplant stress on islets, induces the expression of pro-inflammatory cytokines, thus impairing islet survival and function [8]. Islet transplantation also causes a substantial decrease in oxygen and nutrient delivery to islets by preventing the formation of vascular anastomoses between the islet grafts and the recipient tissue. It has been reported that Insulin-producing pancreatic $\beta$-cells have increased $\beta$-cell death post-transplantation due to increased hypoxic stress that induces apoptosis, leading to $\beta$-cell failure. $\beta$-cells have increased sensitivity to apoptotic signals such as low glucose, hypoxia, cytokines and toxins. Concomitantly, if the expression of cytoprotective anti-apoptotic genes that confer hypoxia-resistance to regular cells are deficient in $\beta$-cells, or are rendered deficient during the isolation process, this could accelerate $\beta$-cell loss.

Low hypoglycemia/hypoxia inducible mitochondrial protein (HIMP1) expression in $\beta$ cells predisposes them to hypoxic injury: Pancreatic $\beta$-cells lack a low glucose and oxygen-induced mitochondrial protein that enhances cell survival [9]. Mitochondria are important cellular organelles that regulate apoptosis in eukaryotic cells. Although it is still unclear that mitochondria are linked to $\beta$-cell apoptotic proneness, the mitochondria are a site of importance for insulin secretion. Gene expression in $\alpha$ and $\beta$-cells was observed to identify their ability to overcome apoptotic stimuli and increase cell survival. HIMP1 (Hypoglycemia/hypoxia Inducible Mitochondrial Protein) is a highly expressed inner mitochondrial membrane protein in pancreatic $\alpha$-cells. Expression of HIMP1 is increased in a low glucose and oxygen environment. Induced gene expression is not seen in $\beta$-cells and could give reason to cells expressing HIMP1 are protected from apoptotic induced stimuli. [10] studied HIMP1 expression in $\alpha$ and $\beta$ cells at various glucose concentrations and discovered that low glucose $(2.5 \mathrm{mM})$ increased HIMP1 expression while high glucose $(25 \mathrm{mM})$ decreased HIMP1 expression [9] in a cells. No expression of HIMP1 was found in $\beta$ cells. However, when HIMP1 was transfected into $\beta$-cells, exposure to low glucose and oxygen levels caused expression of HIMP1 in transfected $\beta$-cells. With no mitochondrial expression of HIMP1, $\beta$-cells demonstrated an increase in mitochondrial apoptotic susceptibility. Thus, it is evident that strategies will need to be developed to protect $\beta$-cells against hypoxic stress post-transplantation and increase oxygen delivery to transplanted islets.

Exposure to hypoxia induces activation of pro-apoptotic intracellular protein complexes in $\beta$ cells: Over time, hypoxia can compromise islet cell function because pancreatic $\beta$-cells are dependent on oxidative metabolism especially at elevated glucose concentrations, as manifested by an increase in their oxygen consumption rate (OCR). Changes in oxygen consumption cause activation of hypoxia-inducible factors (HIFs) in $\beta$-cells and prolong periods of hypoxia compromise islet function post-transplantation. Wang et al. observed that $\beta$ -cell metabolism was accelerated during incubation at high glucose concentrations (16.7 or $33.3 \mathrm{mM}$ ) [9]. OCR measurement is an accurate determinant of islet viability, especially when it is evaluated in response to high glucose stimulation. Increased exposure to hypoxia leads to the downregulation of genes by transcription and inflammatory responses. The nuclear factor kappa-light-chain-enhancer of activated B cells (NFkB) and the metallothionein (MT1/2) gene are both activated by exposure of islets to hypoxia leading to apoptosis [11]. SLC30A8, a gene encoding the zinc transporter 8 (ZnT8), is expressed by $a$ and $\beta$-cells, and is needed in response to zinc accumulation. Hypoxia causes a decrease expression of SLC30A8, leading to reduced ZnT8 expression on the cell membrane, resulting in a drop in cytosolic Zinc concentration by up to $40 \%$, significantly impairing islet survival. After transplantation, regions of the islet graft become non-functional due to hypoxic stress-inducing AMP-activated protein kinases (AMPK). High levels of AMPK cause activation of caspase-3, inducing apoptosis. Thus, one possible strategy would be targeted inhibition of AMPK to prevent apoptosis induction through this pathway, a strategy that has been 
demonstrated to successfully arrest cell death in $\beta$ cell lines exposed to hypoxic culture conditions [12].

Novel strategies to generate hypoxia-resistant islets using genetic modification: Despite improvements in islet transplant outcomes in animal models, roadblocks to the feasibility of clinical islet transplantation still persist. One of the alternative perspectives to solving this problem is genetic modification of islets. Still in its experimental stages, genetically-modified islet represents the fruits of decades of research into the elucidation of the complex intracellular signaling pathways involved in the cellular response to hypoxia. The loss of vascular networks and inadequate oxygen delivery to encapsulated islets leading to islet dysfunction is a tissue-level phenomenon, but specific intracellular developments culminate to upregulate production of reactive oxygen species (ROS), trans-membrane ion-exchangers, and pro-apoptotic genes, all of which contribute to loss of viability at the molecular level. Specifically, modulation of expression levels of certain genes encoding products such as cytoglobin (CYGB), Metallothionein II (MT II), Na-Ca exchanger (NCX1), X-linked inhibitor of apoptosis (XIAP), VEGF, and exendin-4 (Ex-4) have resulted in favorable transplantation outcomes.

Cytoglobin: Cytoglobin has been characterized as an intracellular oxygen scavenging and distribution protein compelling investigators to explore its potential for preservation of islets in hypoxic conditions. Rat islets transfected with a plasmid encoding the CYGB gene displayed superior performance in terms of insulin secretion and resistance to central necrosis compared to control islets in an in vitro hypoxia simulation [13]. Furthermore, when CYGB-transfected rat islets were immune-isolated in a bioartificial pancreas (BAP) composed of polyacrylonitrile-polyvinychloride and implanted under the kidney capsule in diabetic rats, normoglycemia was achieved for $>60$ days, compared to 14 days in the control group [14].

Metallothionein-II (MT-II) and NCX1: The involvement of ROS in hypoxia-related compromise of islet viability has been clearly demonstrated [15]. In an attempt to target ROS up-regulation, islets from transgenic mice established for overexpression of the human Metallothionein-II gene (MT-II), a known anti-oxidant, were transplanted under the kidney capsule of diabetic mice. MT-II islets lowered hyperglycemic indices two times longer on average than wildtype islets, an effect attributed to MT-II mediated protection from extensive free-radical damage conferred upon the transgenic islets [16]. NCX1 has also been a gene of interest due to its involvement in $\beta$-cell proliferation and apoptosis. Utilization of islets from transgenic mice heterozygous for NCX1 implanted under the kidney capsule of diabetic mice produced longer periods of normoglycemia (2-4 times), than wild-type homozygous strains [17].

X-Linked inhibitor of apoptosis protein (XIAP): The manipulation of apoptotic molecular pathways activated by hypoxic conditions has become an approach to enhance islet graft viability compromised in the short-term by intracellular signaling cascades resulting in programmed cell-death. Transduction of the gene encoding XIAP, a ubiquitous inhibitor of apoptosis, into murine $\beta$-cells resulted in resistance to apoptosis following experiments simulating the hypoxic environment of islet transplantation sites [8]. Moreover, XIAP-overexpressing insulin-producing cells implanted subcutaneously in diabetic mice achieved euglycemia in 3 days compared to 21 days [8].

Exendin-4: Investigators have also recently reported the hypoxiamediated apoptosis inhibiting properties of the extendin- 4 protein, a product of the LV-SP-Ex-4 gene [18]. Rat islets transduced with lentiviral vectors containing LV-SP-EX-4 improved islet survival times ( $\sim 18$ days vs. $\sim 10$ days), and decreased the necessary transplant load to induce euglycemia ( 50 islets vs. 150 islets) subsequent to transplantation under the kidney capsule of diabetic mice [19]

Vascular endothelial growth factor (VEGF): Loss of pre-existing microcapillaries supplying islets with adequate oxygen during the isolation process, and delayed angiogenesis in the immediate posttransplant period have been ubiquitously reported as the primary factors precipitating islet graft failure. As such, transduction of the vascular endothelial growth factor (VEGF), noted for its capacity to initiate endothelial cell proliferation, and more importantly islet revascularization in a foreign transplant site [20], emerged as a potential contender to help trigger angiogenesis. Human islets transduced with human-VEGF containing adenoviral vectors resisted caspase-3 activation and apoptosis in vitro through upregulation of antiapoptotic gene expression. Transplantation of $\mathrm{VEGF}^{+}$islets under the kidney capsules of diabetic mice successfully induced lower blood glucose levels, staining positive for insulin [20]. The favorable outcomes of this experiment likely represent VEGF-driven upregulation of vascularization and improved oxygen delivery to islets.

Careful selection of islet Source and transplant site, and hypoxic pre-conditioning can improve transplant outcomes

Islet source: When evaluating alternatives to human islets, porcine islets are believed to have a high potential for clinical translation due to various factors [21]. Donor age, strain and weaning status, and islet maturation and size, have a significant impact on the susceptibility to hypoxia of the xenograft islets. Porcine islets are generally harvested from either neonatal, juvenile or adult pigs, and the choice of one or the other would primarily depend on their ability to respond to in vitro glucose stimulation, to survive during in vitro culture, their ability to proliferate, their antigenicity, and their resistance to hypoxic injury.

Neonatal porcine islets (NPI) have shown to be a promising source of islets for transplantation because NPI exhibit a natural resistance to hypoxia-induced apoptosis as well as hypoxia-reoxygenation insult. Emamaullee et al. reported that unlike adult porcine islets, they are able to recover glucose responsiveness upon recovery from the hypoxic insult. NPI also showed a greater ability to resist apoptosis posttransplantation, and noted to express significantly higher levels of the $\mathrm{X}$-linked inhibitor of apoptosis protein (XIAP) [8].

Role of islet size in transplant outcomes: Choice of islet size also has a significant impact on transplant outcomes as the predisposition to damage by hypoxic conditions. In a rat model, when compared to large islets $(>150 \mu \mathrm{m})$, small islets $(<150 \mu \mathrm{m})$ were more viable in in vitro incubation conditions, showing less necrosis, higher insulin secretion, and greater oxygen consumption. More importantly, upon transplant into diabetic recipients, smaller islets were also able to normalize glycemic levels with greater consistency than large islets [22]. Notably, these results were also confirmed utilizing large and small human islets in regards to viability and insulin secretion in vitro [23]. These findings are manifestations of the relationship between diffusion distance and oxygen bioavailability. In smaller islets, oxygen diffuses readily and is able to reach the center of the islet, resulting in less core necrosis, and greater insulin response overall. Despite the inescapable loss of the vascular anastomoses between the islet and native pancreas microvasculature during the isolation process, small islets are able to sustain insulin-secreting capabilities to greater extent than larger islets [24]. 
Choice of transplant site is a key determinant of prolonged graft success: Encapsulated islets cannot be safely transplanted into the liver through the portal vein, the conventional site of choice in islet transplantation, as the significantly greater graft volume, the geometry and the material of the encapsulant would result in either portal vein occlusion or thrombosis. Thus, other sites like the peritoneal cavity, the omentum, the renal subcapsular space, and the subcutaneous space have all been evaluated as alternatives.

Intraperitoneal islet transplantation: For intraperitoneal transplants, islets are infused into the peritoneal cavity [23]. As an alternative site capable of accommodating such a volumetric load without risk for adverse events, intraperitoneal transplantation of encapsulated islets has achieved transient normoglycemia in diabetic recipients [25,26]; however, most grafts eventually fail due to the physiologic inadequacies of the peritoneum. Despite providing relative ease of access and sufficient space for encapsulated islet transplant, the peritoneal cavity is relatively avascular (compared to the liver, a highly vascular organ), consequently has lower oxygen tension and fails to promote angiogenesis due to free-floating state of capsules in the peritoneal fluid [27]. Another disadvantage of the peritoneal site is that retrieval becomes challenging and there is an increased risk of capsule clumping. The peritoneal cavity is also a hub for sentinel immune cells such as neutrophils, macrophages and fibroblasts that could trigger PCO impeding sufficient oxygen perfusion into the graft [28]. In light of the immunological and physiological challenges peritoneal transplant entails, researchers have explored alternative sites, such as the kidney capsule, and subcutaneous space.

Transplantation into an omental 'pouch': Omental 'pouches' created surgically to serve as an alternative site for islet transplants afford greater retrievability due to ability to concentrate encapsulated islets in a single location. Furthermore, the omentum has proven to be a superior site in a direct comparison to the kidney capsule owing to a more extensive vasculature, portal drainage, volume capacity, and immune-modulation [29]. Nonetheless, the omental pouch also suffers from inadequate oxygen tension to preserve islet viability in the longterm. Microencapsulated murine islets transplanted into the omentum of diabetic mice normalized glycemic indices for extended periods, although eventual detection of necrotic cells, and hyperglycemia was indicative of hypoxic events [30].

Transplantation under the kidney capsule: While it has been reported that the renal subcapsular space can be used for the transplantation of microencapsulated porcine islets [23], the volume of space is limited for the placement of a curative dose of encapsulated islets, especially in the case of human recipients. Nevertheless, when microencapsulated adult porcine islets transplanted into the peritoneal cavity, were compared with those transplanted under the kidney capsule in the rat model, it was clear that capsule integrity was preserved to a greater extent in the kidney (5\% capsule breakage), attributable to restricted mobility of the grafts [31]. PCO was also significantly lower, allowing islets to maintain both viability and functionality [31].

Transplantation into the subcutaneous space: The subcutaneous space allows for easy access and retrieval of implanted islet capsules. However, the subcutaneous space has its own shortcomings such as susceptibility of grafts to mechanical stress, suboptimal vascularization, circulation drainage, and relatively low oxygen tensions without preconditioning [32]. Thus, the subcutaneous site will either require modification, such as the induction of neovascularization, or exogenous oxygen delivery using electrochemical and photosynthetic oxygen generation to increase the chances of transplant success.

Other alternative sites for islet transplantation: A relatively novel site for encapsulated islet transplantation is the bone marrow. In a study evaluating the intramedullary space of diabetic animal models, the bone marrow proved to be a suitable location. Encapsulated islets grafted into the intramedullary space normalized hyperglycemia of diabetic animals for 31 days [33]. This phenomenon was attributed to adequate oxygen tension found in the bone marrow $(40-60 \mathrm{mmHg}$ ), similar to the perfusion intensities in the native environment of pancreatic islet cells [33]. Moreover, the intramedullary space has an extensive circulatory system, which is capable of delivering secreted insulin from the graft instantaneously, as opposed to primary reliance of insulin secreted in the peritoneal cavity, and subcutaneous regions on passive diffusion [33].

The ability of encapsulated islets to restore euglycemia in diabetic animal models tends to vary, with the choice of transplant site wielding a compelling degree of influence on outcome. Despite each site's distinctive advantages and pitfalls, achievement of long-term transplant success has been elusive. A significant portion of graft failure was ultimately attributable to lack of adequate oxygenation of islets removed from their native supply of oxygen supplied at arterial pressures during the isolation process. Oxygen deficiencies are either inherent as a physiological condition of the site itself, a consequence of impeded angiogenesis induced by the presence of a capsular surface preventing islet-host tissue contact, PCO inhibiting gaseous diffusion, or a combination of these factors. Unfortunately, currently no transplant site exists that is both suitable for the increased volumetric load of encapsulated islets, and able to provide oxygen at a tension equivalent to that experienced by native donor islets. To address this problem, pre-conditioning of either the transplant site or the islets themselves via exposure to bioactive compounds or selective pressures prior to implantation is being explored.

Hypoxic pre-conditioning: Since it has been reported that some islets are inherently resistant to hypoxic injury compared to others, researchers have transplanted, retrieved, and isolated sodium alginate LVG-encapsulated rat insulinoma cells into the oxygen-deficient peritoneum of mice, for two cycles, selecting for surviving cells. The viable explanted cells, separated from apoptotic cells and cells having undergone necrosis were evaluated in vitro, and found to be significantly more resistant to hydrogen peroxide, nitric oxide, alloxan, and hypoxia compared to progenitor cells [34]. Researchers also discovered that mouse islets exposed to intermittent hypoxia cycling, specifically for a one-minute period at $5 \%$ oxygen concentration, followed by one minute at $21 \%$ protected islets from extensive decreased glucose response in hypoxic conditions $\left(5 \% \mathrm{O}_{2}\right.$ concentration) [35].

Diazoxide: Islet pre-conditioning through exposure to diazoxide, a potassium channel activator, prior to oxygen deprivation resulted in a smaller decrease in insulin secretion and a $40 \%$ reduction in cell necrosis compared to the extensive functional compromise observed in control islets [36]. Diazoxide pre-conditioned, APA alginatemicroencapsulated rat islets lowered blood glucose levels of diabetic rats by $20 \%$ ( $0 \%$ for unconditioned cells), potentially via activated pathways associated with an increased level of HIF-1 $\alpha$, and phosphorylated AMPK [36].

Desferroxamine: $2.2 \%$ UPMVG alginate-microencapsulated human islets exposed to desferroxamine, an iron chelator, prior to intraperitoneal transplantation into diabetic mice resulted in $100 \%$ normoglycemia restoration as opposed to $36 \%$ attained by unconditioned 
islets at 27 days post-transplantation [37]. Desferroxamine exposure directly correlated with enhanced pro-angiogenic VEGF secretion 1.6-fold by encapsulated islets, and increased HIF-1 $\alpha$ expression, suggesting possible mechanisms by which the iron chelator exerted beneficial effects [37].

Exendin-4: Pre-treatment of rat islets with extendin-4, a glucagonlike peptide-1 receptor agonist molecule with recognized $\beta$-cell antiapoptotic and proliferative effects prior to macroencapsulation and subcutaneous transplant in diabetic mice induced several favorable outcomes. Post-transplant evaluation demonstrated that islet function was maintained ( $100 \%$ in exendin-treated vs. $20 \%$ in control), necrosis curbed, viability preserved, angiogenesis stimulated to greater extent, and ATP levels elevated. Extendin-4 is also hypothesized to exert its promising effects in hypoxic conditions via the upregulation of HIF1a expression, allowing normoglycemia to be achieved at significantly lower doses of encapsulated rat islets (500 IEq, treated; $1000 \mathrm{IEq}$, untreated) [5]. Interestingly, expression of the pro-apoptotic enzyme caspase- 3 was downregulated, and that of the anti-apoptic gene Bcl-2 and Pdx-1 was amplified [5].

Growth hormone-releasing hormone (GHRH) agonist - JI36: Growth hormone-releasing hormone (GHRH) has been shown to protect cardiomyocytes from ischemic injury, and promote angiogenesis by increasing VEGF [38]. GHRH agonist JI-36, a more potent and longer-acting relative, was used to pre-treat rat islets prior to macroencapsulation in $1.8 \%$ high-G alginate, and transplanted subcutaneously into diabetic rats. JI-36 pre-treatment led to a reduction in islet apoptosis, increase in islet proliferation, and decreased the necessary transplant load by half, which could indirectly counter hypoxic degradation in macroencapsulated islets by decreasing cell density [39]. Another study explored the effect of pre-conditioning AN69-membrane encapsulated rat islets with vascular endothelial growth factor (VEGF), prior to immobilization in collagen and transplant into the peritoneal cavity of diabetic mice. Post-explant analysis showed increased angiogenesis, decreased macrophage chemotactic protein concentrations, and decreased cellular adhesion in macrocapsules conditioned with VEGF [40]. Most importantly, a longer duration of normoglycemia achieved in diabetic recipients (25 days vs. 7 days) having received islets exposed to VEGF [40].

Transplant site pre-vascularization: Several researchers have attempted to pre-vascularize subcutaneous sites to counter the native hypoxic environment. De Vos et al. reported that a prevascularized polytetrafluoroethylene (PTFE) 'scaffold' coated with acidic Fibroblast Growth Factor (aFGF) to trigger vascularization, was superior to the unmodified peritoneal cavity as an efficacious transplantation site for encapsulated islets (100\% success, PTFE; $33 \%$ success, unmodified peritoneal cavity) [41]. Luan et al. reported rapid reversal of hyperglycemia after islet transplantation following a one-week period of pre-vascularization using agarose rods infused with basic fibroblast growth factor and heparin. The implanted islet grafts demonstrated long-term viability, as opposed to transient reversion and short-term survival [42]. Pepper et al. reported that using a medically approved vascular access catheter to prevascularize a subcutaneous transplant site, transplantation of 500IEq of syngeneic islets was able to cure $91 \%$ of diabetic mouse recipients for $>100$ days [43]. These studies and others clearly demonstrate the importance of transplant site prevascularization in achieving hyperglycemia reversal by mitigating hypoxia in transplanted islet grafts, especially at transplant sites where the unmodified environment is poorly vascularized.

\section{Optimization of bioencapsulation device parameters can improve islet transplant outcomes}

The bioartificial pancreas (BAP) has been developed to regulate and control pancreatic islet $\beta$-cell transportation of oxygen, glucose, nutrients, metabolites, and waste. However, BAP limits diffusive transfer of oxygen, and hence islet oxygen consumption, which leads to chronic $\beta$-cell hypoxia. Microencapsulaton of $\beta$-cells has primarly focused on two kinds of materials - thermoplastic polymers and hydrogel polymers. Hydrogels have become the most widely used for microencapsulation because they provide substantial diffusive properties. Thermopolymers such as hydrolytically active polydimethylsiloxane (PDMS) have been used for microencapsulation but have limitations in diffusive properties of water-soluble nutrients. Since PDMS is hydrophobic, the polymer restricts the diffusion of water through the biomaterials, thus impeding solute and water-soluble nutrient diffusion.

Effect of microencapsulation on islet oxygenation: Islet encapsulation results in $\beta$-cell death during the early post-transplantation period by worsening tissue hypoxia by increasing the diffusion distance to the islet core, especially when compared to non-encapsulated islets. While several research groups have reported greater incidence of hypoxic injury in encapsulated islets, Hals IK et al. reported that while alginate-encapsulated islets demonstrated a significant reduction in viability $(33.8 \pm 3.5 \%$, encapsulated; $42.9 \pm 5.2 \%$; non-encapsulated islets), basal oxygen consumption was reduced equally for both groups for encapsulated and non-encapsulated islets $(22.0 \pm 6.1 \%$ versus $24.8 \pm$ 5.7\%). Thus, microencapsulated human islets were showed no increase of susceptibility to hypoxia [44]. Schrezenmeir et al. reported that barium alginate microencapsulation reduced islet oxygenation due to the expansion of an unstirred water layer surrounding tissue. Oxygen tensions $\left(\mathrm{pO}_{2}\right)$ measured using a microelectrode showed a value of $44 \pm$ $5 \%$ for encapsulated tissue compared with $64 \pm 4 \%$ in nonencapsulated tissue. Additionally, oxygen tension in the islet core dropped to $11 \pm 3 \%$ for encapsulated tissue compared with $22 \pm 4 \%$ for nonencapsulated tissue, thus illustrating that barium alginate encapsulation may actually worsen oxygenation [45]. It is noteworthy that no similar studies were performed with calcium alginate microcapsules, thus it is uncertain whether they would behave similar to barium alginate.

Optimization of microcapsule size and volume results in improved transplant outcomes: Alginate has become the most widely studied polymer for islet microencapsulation. Alginate is extracted from brown algae, and is composed of a polysaccharide chain containing mannuronic acid (M-blocks), guluronic acid (G block) and mixed MG-blocks. Alginate is a non-toxic hydrogel that functions as an immunoprotective barrier to the donor islet grafts. Microcapsules have to be able to withstand physical and osmotic stress to avoid capsule breakage and immune-mediated rejection. The ideal capsule should allow unimpeded diffusion of glucose and insulin while preventing immune cell infiltration of the alginate barrier; capsule size should be small with graft volume kept to a minimum. It is self-evident, that microcapsule volume is a function of its radius to the power of three i.e. if microcapsule size is halved, the volume will decrease to an eighth [22]. Optimization of microcapsule size can improve insulin secretion and nutrient uptake by the islets within. Very small capsules can undergo increased osmotic stress by swelling. However, very large capsules have larger diffusion distances for glucose and oxygen to reach the islet core. Microcapsule size can be controlled using a high voltage electrostatic bead generator [46]. Changing the voltage, needle diameter, distance between the needle and gel solution, and flow rate, could all be used to control the size of capsules. 
Macroencapsulation device geometry can be tailored to minimize islet hypoxia: Dulong et al. immobilized islets in hollow fibers at varying densities to determine the effects of fiber geometry on islet necrosis and hypoxia [47]. To determine fiber geometry efficiency, a mathematical model observed fiber geometry of capsules to demonstrate efficacy of insulin secretion and necrosis ratio by differing fiber length and diameter. High-density fibers demonstrated a larger necrosis ratio and fiber radius demonstrated that the higher the density of islets, the shorter the fiber, but the larger the number of islets required. A shorter fiber could reduce the fiber length but a larger radius and increase number of islets is needed [47]. Cylindrical geometry demonstrated low incidence of islet necrosis (13\%) but the fibers were impractically long for islet seeding [47]. Since modeling predicted that 270 such fibers would require to be implanted, their unusual length would make it impossible. To ensure complete oxygenation of islets, the fibers must be spread far apart. Flat sheet geometry demonstrated an increase in the number of islets in order for the islets to release an appropriate amount of insulin with higher density and thicker fibers. It was also noted that flat geometry seemed to cause problems for implantation as a robust foreign body response caused the geometry of the implant to revert to the form of a 'bowl'.

Although microencapsulation has been very successful in various areas of biotechnology, it has consistently failed in humans. The alginate microcapsules have a high propensity to aggregate, severely limiting oxygen uptake.

Macroencapsulation device surface roughness promotes angiogenesis: An alternative approach to conventional microencapsulation is the use of 'capillaries' for macroencapsulation because capillaries have a greater surface-area-to-volume ratio and can be refilled. Capillaries made out of hydroxymethylated polysulfone with a rough and open-porous outer surface triggered the rat (or porcine) islets within to release VEGF, stimulating angiogenesis, thus prolonging islet function in vitro [48]. When these encapsulated islets were transplanted into diabetic rats, the recipient animals demonstrated a reduction in blood glucose levels during the first week after transplantation. When capillaries containing rat (or porcine) islets were explanted from normoglycemic rats, they showed signs of vascularization [48]. Transplanted capillaries with no islets had no vascularization. These observations demonstrate that islets encapsulated within hydroxymethylated polysulfone 'capillaries' release VEGF that allows for vascularization and prolonged islet function posttransplantation.

\section{Co-encapsulation of islets with angiogenic agents, oxygen transporters and supportive cell types}

The presence of a capsule layer surrounding the islets creates an unfavorable diffusive gradient, impedes angiogenesis, eliminates the possibility of certain transplant sites, and may promote PCO, all of which further exacerbate hypoxia related damage to islets. However, a semi-porous membrane, if actively manipulated to secrete certain bioactive molecules or utilized to co-encapsulate potentially complementary cells, simultaneously protecting and holding the two types of cells in close proximity, could actually prove beneficial as an essential component of an alternate perspective on hypoxia alleviation. In particular, adjusting the structure of the bioartifical pancreas to hold, and secrete pro-angiogenic proteins, incorporating cross-linked hemoglobin or perfluorocarbons, and co-encapsulation of islets with mesenchymal stem cells (MSCs) will be further explored in this section.

Angiogenic agents - fibroblast growth factor - 1: Fibroblast
Growth Factor - 1 (FGF-1) has been characterized widely in the literature as a pro-angiogenic protein, and its ability to induce neovascularization in the rat omentum, a common site for encapsulated islet transplants, has been evaluated. High-G, low-viscosity, $\mathrm{Ca}^{++}$crosslinked alginate microcapsules encapsulated with FGF-1 were found to induce greater vascular density compared with control microcapsules [49]. McQuilling et al. demonstrated that when modified $\sim 600 \mu \mathrm{m}$ poly-L-ornithine (PLO) grafted, $\mathrm{Ca}^{+}{ }^{+}$cross-linked alginate microcapsules, were loaded with human FGF-1 into the external layer and transplanted into the omentum of rats, the mean vessel density was higher in FGF-1 loaded microcapsules (198.8 mm2, FGF-1; $128.9 \mathrm{~mm} 2$, control) [50]. When encapsulated rat islets in the $1.5 \%$ LVM alginate inner core, along with FGF-1 in the outer layer composed of $1.25 \%$ LVG (separated by a layer of PLO) were transplanted into the omental pouch of diabetic rats, both the FGF-1 containing, and control grafts were essentially equivalent in terms of lowering hyperglycemia in the recipients [51]. However, it is believed that this could be attributable to the well-vascularized nature of the omentum, and the neovascularization promoted by encapsulation of FGF-1 could prove more beneficial in less perfused sites such as the peritoneum or subcutaneous space.

Hemoglobin crosslinking: Having been removed from the pancreatic microvasculature allowing for direct diffusion of oxygen from the arterial blood $\left(\mathrm{pO}_{2} \sim 100 \mathrm{mmHg}\right)$, microencapsulated islets, isolated from their native environment, and reliant solely on gaseous diffusion are extremely susceptible to hypoxia. For this reason, dissolved hemoglobin $(\mathrm{Hb})$, has been examined for its capabilities to improve functionality and viability of macroencapsulated islets cultured in vitro [52]. Chae et al. modified Hemoglobin by crosslinking it to a watersoluble polymer with a sufficient molecular weight $(100 \mathrm{kDa})$ to keep it from diffusing out of microcapsule pores. To increase protection against leakage, a non-traditional, 5-layer alginate-PLL-alginate-PLLalginate microcapsule was synthesized. Islets insulated by capsules that incorporate crosslinked hemoglobin $(\mathrm{Hb}-\mathrm{C})$ into the membrane via electrostatic interactions showed less central necrosis, and increased insulin secretion compared with the control group [53]. This difference became even more pronounced at high glucose concentrations (typical of a diabetic animal model), where oxygen demands on the islet are exhaustive, and graft cores have a greater propensity to undergo necrosis as peripheral cells consume available oxygen supplies [54]. Interestingly, in addition to the facilitation of oxygen transport, the capacity of $\mathrm{Hb}-\mathrm{C}$ to scavenge reactive oxygen species (ROS) has been suggested as one of the reasons viability and functionality of islets were preserved in the treatment group [54]. The production of ROS such as nitric oxide has been known to be upregulated in islets during hypoxic conditions leading to apoptosis and necrosis [55].

Perfluorocarbon emulsions mitigate hypoxic injury in encapsulated islets: New methods have recently been developed to improve microencapsulated islet viability since encapsulation has been shown to be detrimental to adequate $\beta$-cell oxygenation. Barium alginate and barium alginate containing $70 \%$ perfluorocarbon (PFC) emulsion microcapsules were evaluated to assess their effect on islet OCR. PFC emulsions made from perfluorodecalin and 20\% Intralipid (Baxter) were encapsulated with low viscosity high glucuronate (LVG) alginate to make PFC capsules. After exposure to low oxygen levels for 48 hours, islets encapsulated in alginate had reduced viability and displayed necrosis. However, alginate co-encapsulated with PFC had maintained oxygen consumption rates and little necrosis was observed. By incorporating PFC emulsions into alginate microcapsules, microcapsule oxygen permeability is increased and islet survival is preserved even under hypoxic conditions. However, 
at high concentrations, PFC is toxic to the islets themselves, leading to a reduction in islet viability. Other disadvantages include loss of microcapsule transparency, which renders assays to assess islet function and viability impossible to perform accurately [56].

Mesenchymal stem cells (MSCs): Mesenchymal stem cells (MSCs) have been known to promote wound-healing, cell-survival, and have even been reported to secrete VEGF in vitro [57,58]. Therefore, investigators have explored the potential advantages of employing them as a supplementary tool to augment resistance to hypoxic injury in islets. In in vitro conditions, rat islets co-cultured with MSCs exhibited increased expression of anti-apoptotic genes such as HIF-1 1 , HO-1, and COX-2, and enhanced glucose-stimulated insulin secretion, unlike control islets [59]. Similarly, Qin et al. reported a lower ADP/ATP ratio in islets co-cultured with human MSCs, indicative of increased resistance to necrosis and apoptosis, maintenance of glucose-stimulated insulin secretion, increase in expression of anti-apoptotic proteins, including Bcl-xL, Bcl-2, HSP-32, and XIAP. Notably, an increase in the gene expression of VEGF-receptor 2 (VEGFR2), and an increase of pro-angiogenic factors was observed in the MSC-co cultured islets [57]. In vivo outcomes substantiated prior findings, as diabetic mice transplanted with MSC pre-treated islets experienced better glycemic control and improved blood vessel formation at the transplant site compared to controls [57].

Immunoisolating islets using encapsulation presents a compelling opportunity to co-encapsulate MSCs with islets as this proximity could directly improve islet function and survival mediated by secreted cytoprotective biomolecules. It was observed that when encapsulated porcine islets were transplanted subcutaneously into diabetic rats, they exhibited reduced islet oxygenation when compared with empty capsules implanted in non-diabetic rats. However, when adiposederived mesenchymal cells (AMSCs) were co-encapsulated with these islets, they were able to counter hypoxia exacerbated by hyperglycemia via VEGF-mediated neovascularization [60]. When porcine MSCs were co-encapsulated with porcine islets and transplanted subcutaneously in diabetic rats and primates, angiogenesis was stimulated, and graft oxygenation improved in the short term (1-4 weeks) [58]. However, introduction of MSCs in macrocapsules with islets failed to produce significant enhancement of graft survival and function in the long-term (> 4 weeks), regardless of persistent blood vessel proliferation; here, other factors such as suboptimal macrocapsule size and geometry and poor immunoisolation might have interfered with graft survival [58].

\section{Strategies aimed at supplementing oxygen to encapsulated islets}

The $\beta$-air device: The $\beta$-Air device was designed as a subcutaneous implant to enhance oxygen supply and protect donor islets against the host immune system. The device is minimally invasive and can be refueled with oxygen through an implanted access port [61]. It contains a bioreactor with an islet module containing encapsulated islets in an alginate gel and a gas chamber separate from the islet module. The design of the islet module is flat to create a uniform spread of islet oxygenation.

The device has been evaluated in several small and large animal trials; when implanted in streptozotocin-induced diabetic rats, they were able to reverse hyperglycemia for up to 6-months, so long as a steady supply of oxygen was available; Oxygen is contained in a chamber that can diffuse across a gas-permeable membrane and supply encapsulated islets with oxygen. To sustain islet viability and avoid hypoxic stress, the gas chambers had to be replenished daily with a gas mixture of $20-30 \%$ oxygen. Observations indicated that the $\beta$-Air could maintain donor islet function with an islet density of $\sim 1,000 \mathrm{IEQ} / \mathrm{cm} 2$, for 180 days with atmospheric air. The $\beta$-Air II (a newer prototype) needed oxygen refueling after a 24 -hour period, which suggested that $60-75 \%$ of oxygen was taken up by the islet graft and remaining oxygen diffused into surrounding host tissue [61]. Both devices had normal glycemic indicators upon implantation and maintained normalized glycemic levels up to 180 days.

In another study, an allogeneic pig model used macroencapsulated porcine islets with $2.5 \%$ concentrated guluronic acid alginate crosslinked with $70 \mathrm{mM} \mathrm{SrCl}$. Macroencapsulated porcine islets (3000 IEQ) were implanted within the $\beta$-Air device and the device was implanted subcutaneously in the lateral abdomen of female Göttingen Minipigs [62]. After 13 days post-transplantation, the device was explanted and analyzed for morphological appearance and glucose-stimulated insulin release (GSIR) profile. Histology of the device showed no signs of inflammatory response or fibrotic reaction [62]. Islet morphology was maintained and the $\beta$-cell mass remained unchanged. While insulin release (GSIR) prior to implantation demonstrated a stimulation index of 3.5, after explantation, the stimulation index was 6.7. [62]. Transplantation of allogeneic pig islets into female Göttingen Minipigs using the $\beta$-Air, did not require the use of immunosuppressant drugs. The implanted device was able to maintain biocompatibility and physiological concentrations of oxygen. Glycemic control was normalized, suggesting the ability of $\beta$-Air to maintain long-term normalization of insulin secretion in allogeneic diabetic models.

Photosynthetic oxygen generation: An alternative approach for co-encapsulation of islets in alginate is to use a photosynthetic oxygen generator. A strain of unicellular algae Chlorella spp., was used as a photosynthetic oxygen generator to provide oxygenation to encapsulated islets. Photosynthetic Chlorella spp. can generate oxygen by using $\mathrm{CO}_{2}$ to stimulate insulin secretion by encapsulated islets. Under severe hypoxic conditions, Chlorella can compensate for lack of oxygen in an oxygen-free medium by generating oxygen to sustain islet function. Bloch et al. reported that when islets isolated from male ICR mice were encapsulated with and without Chlorella spp. to observe insulin release (GSIR) and oxygen generation (using a Clark electrode chamber) the photosynthetic algae provided a substantial oxygen supply for the islets [63]. Using photosynthetic algae for the bioartificial pancreas may eliminate the need to charge the device with a continuous $20-30 \%$ oxygen gas mixture. However, continuous light transmission will be required to optimize the production of oxygen by photosynthetic algae. Before long-term application of photosynthetic microcapsules can be used to enhance the bioartificial pancreas, further approaches need to be taken to understand transfer limitations due to fibrosis, matrix stability, illumination, and adequate energy supply.

Use of oxygen-releasing chemical agents: Several peroxide and percarbonate compounds have been known for decades to be able to produce a steady stream of oxygen when exposed to water. This principle was used by Pedraza et al. when they encapsulated PDMS with solid calcium peroxide (PDMS- $\mathrm{CaO}_{2}$ ) to create an oxygengenerating biomaterial that can generate oxygen for a 6-week period $\left(0.026 \mathrm{mM}\right.$ per day). The presence of PDMS- $\mathrm{CaO}_{2}$ was able to reduce $\beta$-cell hypoxic stress and increase $\beta$-cell viability for a week even under hypoxic conditions. With the encapsulation of solid calcium peroxides in the device, the calcium peroxide reacts by hydration to break down into oxygen. Encapsulation allows for slow oxygen release by diffusion across a semi-permeable barrier. The hydrophobic PDMS biomaterial controls reactivity, increases the generation of oxygen production to oxygenate $\beta$-cells, and decreases hydroxyl radicals destroying $\beta$-cells. 
Removal of PDMS encapsulation causes calcium-peroxide to rapidly release oxygen to its surrounding environment once in contact with water. The result is bursts of oxygen and the generation of hydroxyl radicals that cause severe $\beta$-cell damage. Encapsulation of calciumperoxide drastically reduces reactivity.

When PDMS- $\mathrm{CaO}_{2}$ disks were co-cultured with mouse insulinoma 6 (MIN6) $\beta$-cells and pancreatic rat islets, hypoxia induced stress and death was dramatically reduced. MIN6 $\beta$-cell and pancreatic rat islet survival was monitored for a 3 -week period. On removing PDMS$\mathrm{CaO}_{2}$ microcapsules, treated groups reverted to reduced survival due to increased hypoxic stress [64]. Since PDMS- $\mathrm{CaO}_{2}$ disks rely on high cell loading densities, they could be ideal for tissue oxygenation posttransplantation. This method could be invaluable to ensure islet survival during the initial engraftment period to reduce hypoxia-induced graft loss early after post-transplantation.

Hyperbaric oxygen therapy (HBO): Hypoxia induces islets to

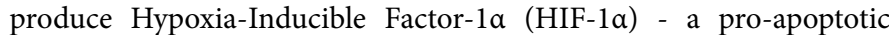
transcription factor and a marker used to evaluate hypoxic responses in early-transplanted islets. Hyperbaric oxygen therapy (HBO) has been suggested to help suppress HIF-1 $1 \alpha$ expression and prevent early $\beta$-cell death during transplantation. When hyperbaric oxygen (HBO) therapy was provided for a 3-day period, HIF-1 $a$ expression was suppressed, improving islet function and reducing graft failure [3]. Maio et al. reported that when islets (treated with $\mathrm{HBO}$ and untreated control) transplanted under the kidney capsule were recovered at day $1,3,7$, and 14 post-transplant, HIF-1a expression was seen in in day 1 and day 3 specimens whereas day 7 specimens had less HIF-1a expression. By day 3 , insulin secretion had dropped $41 \%$ (compared to day 1 ), but mildly rose by day 7 , and increased $54 \%$ and $37 \%$ by day 14 compared to days 3 and 7. Early islet grafts evaluated for hypoxia by TUNEL staining and for apoptosis using BNIP3 expression demonstrated increased BNIP3 expression on days 1 and 3 compared to days 7 and 14 [3]. Higher levels of HIF-1a were observed in early post-transplanted grafts compared to grafts left longer in the kidney capsule. As HIF-1a expression decreased, insulin expression was able to recover compared to early grafts. It is thus postulated that early suppression of HIF-1 $\alpha$ may benefit islet survival.

In order to counter hypoxic injury in encapsulated islets, $\mathrm{HBO}$ was employed to trigger implant site pre-vascularization. This increased vessel growth and VEGF levels and lowered the proportion of encapsulated islets exhibiting hypoxia-induced apoptosis in vivo [65]. HBO therapy was studied further to investigate its efficacy in hypoxia amelioration and improving islet graft survival [66]. Islets were transplanted into the portal vein into the diabetic Balb/c mice at a dose of 500 IEQ per recipient. The islets were either exposed to HBO treatment with $100 \%$ oxygen for 1 hour at 2.5 atmospheres absolute or left untreated. Mice that received $\mathrm{HBO}$ treatment for 6-days, twice daily demonstrated a significant reduction in HIF-1 $\alpha$, TUNEL, and VEGF expression in islets 7 days after transplantation. Histological findings of transplanted islets and surrounding liver tissue revealed severe hypoxic stress, apoptosis, and necrosis by day 3. By day 7, islet apoptosis and necrosis were reduced in HBO-treated islets (but not in controls) and by day 14, demonstrated return to normoxic conditions with no apoptosis or necrosis. HBO-treated mice showed significantly higher levels of plasma insulin. Nearly half of the mice that received HBOtreated islets $(n=9)$ and less than a third of those that received untreated islets $(n=10)$ returned to normoglycemia [66].

These studies demonstrate $\mathrm{HBO}$ as a possible therapeutic option in treating islets in order to avoid acute hypoxic stress and early onset of apoptosis post-transplantation. Mechanisms of $\mathrm{HBO}$ may relate to reduction of tissue hypoxia by decreasing cytokine levels and inhibition of pro-apoptotic induced signaling factors. HBO treated groups demonstrated a decrease in islet hypoxia and apoptosis posttransplantation compared to control groups. HBO Treatment reduced VEGF expression by day 7 post-transplant and serum insulin production was significantly higher in the treated groups. $\mathrm{HBO}$ treatment has the potential to reduce islet hypoxic stress and improve islet function in the recipient. Dynamic Contrast-Enhanced Magnetic Resonance Imaging (DCE MRI) can be used to monitor post-transplant neovascularization to observe vascular maturation after therapy. The technique is noninvasive and can further improve therapeutic treatment using HBO.

\section{Conclusion}

Clinical success of islet transplantation is currently still hampered a combination of insufficient supply of human donors, immunosuppression requirement, and lack of pre-existing vascular oxygen supply. However, various methods have been proposed and tested with promising results to provide alternative sources of islets, reducing or eliminating immunuosuppression requirement, and/ or protecting the transplanted islets against initial hypoxia mediated cell death. The methods include barriers that provide immunoisolating properties, treatments that improve initial oxygenation of the transplants, modifications that promotes angiogenesis, as well as genetic modification that promise to protect transplanted islets from hypoxia. These methods hold the potential to allow for a more wide utilization of islet transplantation that has reduced or eliminated requirement for life-long immunosuppression.

\section{References}

1. PREVENTION, C.F.D.C.A., 2014. National Diabetes Statistics Report Estimates of Diabetes and Its Burden in the United States, 2014. In: Prevention, C.f.D.C.a. (Ed.). US Department of Health and Human Services, Atlanta, GA.

2. Guariguata L, Whiting DR, Hambleton I, Beagley J, Linnenkamp U, et al. (2014) Global estimates of diabetes prevalence for 2013 and projections for 2035 Diabetes Res Clin Pract 103: 137-149.

3. Miao G, Ostrowski RP, Mace J, Hough J, Hopper A, et al. (2006) Dynamic production of hypoxia-inducible factor-1alpha in early transplanted islets. Am J Transplant 6: 2636-2643

4. Ko SH, Ryu GR, Kim S, Ahn YB, Yoon KH, et al. (2008) Inducible nitric oxide synthase-nitric oxide plays an important role in acute and severe hypoxic injury to pancreatic beta cells. Transplantation 85: 323-330.

5. Jia X, Sharma A, Kumagai-Braesch M, Wernerson AM, Sörenby AK, et al. (2012) Exendin- 4 increases the expression of hypoxia-inducible factor- $1 \hat{I} \pm$ in rat islets and preserves the endocrine cell volume of both free and macroencapsulated islet grafts. Cell Transplant 21: 1269-1283.

6. Goto M, Imura T, Inagaki A, Ogawa N, Yamaya H, et al. (2010) The impact of ischemic stress on the quality of isolated pancreatic islets. Transplant Proc 42 2040-2042.

7. Olsson R, Carlsson PO (2011) A low-oxygenated subpopulation of pancreatic islets constitutes a functional reserve of endocrine cells. Diabetes 60: 20682075.

8. Emamaullee JA, Shapiro AM, Rajotte RV, Korbutt G, Elliott JF (2006) Neonata porcine islets exhibit natural resistance to hypoxia-induced apoptosis. Transplantation 82: 945-952.

9. Wang W, Upshaw L, Strong DM, Robertson RP, Reems J (2005) Increased oxygen consumption rates in response to high glucose detected by a nove oxygen biosensor system in non-human primate and human islets. J Endocrinol 185: $445-455$.

10. Wang J, Cao Y, Chen Y, Chen Y, Gardner P, et al. (2006) Pancreatic beta cells lack a low glucose and O2-inducible mitochondrial protein that augments cell survival. Proc Natl Acad Sci U S A 103: 10636-10641.

11. Lai Y, Brandhorst H, Hossain H, Bierhaus A, Chen C, et al. (2009) Activation of 
Citation: Krishnan R, Ko D, Tucker T, Opara E, Foster CE, et al. (2016) Strategies to Combat Hypoxia in Encapsulated Islet Transplantation. Surgery Curr Res 6: 259. doi:10.4172/2161-1076.1000259

Page 9 of 10

NFÎ̊ ${ }^{\circ}$ dependent apoptotic pathway in pancreatic islet cells by hypoxia. See comment in PubMed Commons below Islets 1: 19-25.

12. Ryu GR, Lee MK, Lee E, Ko SH, Ahn YB, et al. (2009) Activation of AMPactivated protein kinase mediates acute and severe hypoxic injury to pancreatic beta cells. Biochem Biophys Res Commun 386: 356-362.

13. Stagner J, Seelan RS, Parthasarathy RN, White K (2009) Reduction of ischemic cell death in cultured Islets of Langerhans by the induction of cytoglobin. Islets 1: $50-54$

14. Stagner JI, Seelan RS, Parthasarathy RN (2011) Maintenance of aerobic metabolism increases immunoisolated islet survival. Islets 3: 89-92.

15. Davalli AM, Scaglia L, Zangen DH, Hollister J, Bonner-Weir S, et al. (1996) Vulnerability of islets in the immediate posttransplantation period. Dynamic changes in structure and function. Diabetes 45: 1161-1167.

16. Li X, Chen H, Epstein PN (2004) Metallothionein protects islets from hypoxia and extends islet graft survival by scavenging most kinds of reactive oxygen species. J Biol Chem 279: 765-771.

17. Nguidjoe E, Sokolow S, Bigabwa S, Pachera N, D'Amico E, et al. (2011) Heterozygous inactivation of the $\mathrm{Na} / \mathrm{Ca}$ exchanger increases glucose-induced insulin release, $\hat{l}^{2}$-cell proliferation, and mass. Diabetes 60: 2076-2085.

18. Ah Kim H, Lee S, Park JH, Lee S, Lee BW, et al. (2009) Enhanced protection of Ins-1 beta cells from apoptosis under hypoxia by delivery of DNA encoding secretion signal peptide-linked exendin-4. J Drug Target 17: 242-248.

19. Jeong JH, Yook S, Jung Y, Im BH, Lee M, et al. (2012) Functional enhancement of beta cells in transplanted pancreatic islets by secretion signal peptide-linked exendin-4 gene transduction. Journal of controlled release : official journal of the Contzrolled Release Society 159: 368-375

20. Mahato RI (2009) Gene expression and silencing for improved islet transplantation. J Control Release 140: 262-267.

21. Ellis CE, Korbutt GS1,2 (2015) Justifying clinical trials for porcine islet xenotransplantation. Xenotransplantation 22: 336-344.

22. MacGregor RR, Williams SJ, Tong PY, Kover K, Moore WV, et al. (2006) Smal rat islets are superior to large islets in in vitro function and in transplantation outcomes. Am J Physiol Endocrinol Metab 290: E771-779.

23. Lehmann R, Zuellig RA, Kugelmeier P, Baenninger PB, Moritz W, et al. (2007) Superiority of small islets in human islet transplantation. Diabetes 56: 594-603.

24. Menger MD, Jaeger S, Walter P, Feifel G, Hammersen F, et al. (1989) Angiogenesis and hemodynamics of microvasculature of transplanted islets of Langerhans. Diabetes 38 Suppl 1: 199-201.

25. Foster JL1, Williams G, Williams LJ, Tuch BE (2007) Differentiation of transplanted microencapsulated fetal pancreatic cells. Transplantation 83 : $1440-1448$.

26. Rayat GR, Rajotte RV, Ao Z, Korbutt GS (2000) Microencapsulation of neonatal porcine islets: protection from human antibody/complement-mediated cytolysis in vitro and long-term reversal of diabetes in nude mice. Transplantation 69 : 1084-1090.

27. Köllmer M, Appel AA1, Somo SI, et al. (2015) Long-Term Function of AlginateEncapsulated Islets. Tissue Eng Part B Rev

28. Vaithilingam V, Fung C, Ratnapala S, Foster J, Vaghjiani V, et al. (2013) Characterisation of the xenogeneic immune response to microencapsulated fetal pig islet-like cell clusters transplanted into immunocompetent C57BL/6 mice. PLoS One 8: e59120.

29. Scharp DW, Swanson CJ, Olack BJ, Latta PP, Hegre OD, et al. (1994) Protection of encapsulated human islets implanted without immunosuppression in patients with type I or type II diabetes and in nondiabetic control subjects. Diabetes 43: 1167-1170.

30. Kobayashi T, Aomatsu Y, Iwata H, Kin T, Kanehiro H, et al. (2006) Survival of microencapsulated islets at 400 days posttransplantation in the omental pouch of NOD mice. Cell Transplant 15: 359-365.

31. Dufrane D, Steenberghe Mv, Goebbels RM, Saliez A, Guiot Y, et al. (2006) The influence of implantation site on the biocompatibility and survival of alginate encapsulated pig islets in rats. Biomaterials 27: 3201-3208.

32. Bartholomeus K, Jacobs-Tulleneers-Thevissen D, Shouyue S, Suenens K, In't Veld PA, et al. (2013) Omentum is better site than kidney capsule for growth, differentiation, and vascularization of immature porcine $\hat{\imath}^{2}$-cell implants in immunodeficient rats. Transplantation 96: 1026-1033.

33. Yang KC, Wu CC, Kuo ZF, Yang CY, Lin FH (2010) Intramedullary cavity as implantation site for bioartifical pancreas: preliminary in vivo study. Transplant Proc 42: 2666-2668

34. Bloch K1, Bloch O, Tarasenko I, Lazard D, Rapoport M, et al. (2011) A strategy for the engineering of insulin producing cells with a broad spectrum of defense properties. Biomaterials 32: 1816-1825.

35. Lo JF, Wang Y, Blake A, Yu G, Harvat TA, et al. (2012) Islet preconditioning via multimodal microfluidic modulation of intermittent hypoxia. Anal Chem 84 1987-1993.

36. Ma Z, Moruzzi N, Catrina SB, Hals I, Oberholzer J, et al. (2013) Preconditioning with associated blocking of $\mathrm{Ca} 2+$ inflow alleviates hypoxia-induced damage to pancreatic $\hat{I}^{2}$-cells. PLoS One 8: e67498.

37. Vaithilingam V, Oberholzer J, Guillemin GJ, Tuch BE (2010) Beneficial effects of desferrioxamine on encapsulated human islets--in vitro and in vivo study. Am J Transplant 10: 1961-1969.

38. Ludwig B, Zimerman B, Steffen A, Yavriants K, Azarov D, et al. (2010) A nove device for islet transplantation providing immune protection and oxygen supply. Horm Metab Res 42: 918-922.

39. Ludwig B, Rotem A, Schmid J, Weir GC, Colton CK, et al. (2012) Improvement of islet function in a bioartificial pancreas by enhanced oxygen supply and growth hormone releasing hormone agonist. Proc Natl Acad Sci U S A 109 : 5022-5027.

40. Sigrist S, Mechine-Neuville A, Mandes K, Calenda V, Braun S, et al. (2003) Influence of VEGF on the viability of encapsulated pancreatic rat islets after transplantation in diabetic mice. Cell Transplant 12: 627-635

41. De Vos P, Hillebrands JL, De Haan BJ, Strubbe JH, Van Schilfgaarde R (1997) Efficacy of a prevascularized expanded polytetrafluoroethylene solid support system as a transplantation site for pancreatic islets. Transplantation 63: 824830

42. Luan NM, Iwata H (2014) Long-term allogeneic islet graft survival in prevascularized subcutaneous sites without immunosuppressive treatment Am J Transplant 14: 1533-1542.

43. Pepper AR1, Gala-Lopez B1, Pawlick R1, Merani S1, Kin T2, et al. (2015) A prevascularized subcutaneous device-less site for islet and cellular transplantation. Nat Biotechnol 33: 518-523.

44. Hals IK, Rokstad AM1, Strand BL2, Oberholzer J3, Grill V4 (2013) Alginate microencapsulation of human islets does not increase susceptibility to acute hypoxia. J Diabetes Res 2013: 374925.

45. Schrezenmeir J, Velten F, Beyer J (1994) Immobilized hemoglobin improves islet function and viability in the bioartificial pancreas in vitro and in vivo. Transplant Proc 26: 792-800.

46. Orive G, Hernández RM, Rodríguez Gascón A, Calafiore R, Chang TM, et al (2004) History, challenges and perspectives of cell microencapsulation. Trends Biotechnol 22: 87-92.

47. Dulong JL, Legallais C (2007) A theoretical study of oxygen transfer including cell necrosis for the design of a bioartificial pancreas. Biotechnol Bioeng 96 990-998.

48. Lembert N, Wesche J, Petersen P, Doser M, Zschocke P, et al. (2005) Encapsulation of islets in rough surface, hydroxymethylated polysulfone capillaries stimulates VEGF release and promotes vascularization after transplantation. Cell Transplant 14: 97-108.

49. Moya ML, Garfinkel MR, Liu X, Lucas S, Opara EC, et al. (2010) Fibroblast growth factor-1 (FGF-1) loaded microbeads enhance local capillary neovascularization. J Surg Res 160: 208-212.

50. McQuilling JP, Arenas-Herrera J, Childers C, Pareta RA, Khanna O, et al (2011) New alginate microcapsule system for angiogenic protein delivery and immunoisolation of islets for transplantation in the rat omentum pouch. Transplant Proc 43: 3262-3264

51. Pareta R, McQuilling JP, Sittadjody S, Jenkins R, Bowden S, et al. (2014) Long term function of islets encapsulated in a redesigned alginate microcapsule construct in omentum pouches of immune-competent diabetic rats. Pancreas 43: $605-613$.

52. Schrezenmeir J1, Kirchgessner J, Gerö L, Kunz LA, Beyer J, et al. (1994) Effect of microencapsulation on oxygen distribution in islets organs. Transplantation 
Citation: Krishnan R, Ko D, Tucker T, Opara E, Foster CE, et al. (2016) Strategies to Combat Hypoxia in Encapsulated Islet Transplantation. Surgery Curr Res 6: 259. doi:10.4172/2161-1076.1000259

Page 10 of 10

\section{7: $1308-1314$}

53. Chae SY1, Kim SW, Bae YH (2002) Effect of cross-linked hemoglobin on functionality and viability of microencapsulated pancreatic islets. Tissue Eng 8: 379-394.

54. Carlsson PO, Liss P, Andersson A, Jansson L (1998) Measurements of oxygen tension in native and transplanted rat pancreatic islets. Diabetes 47: 10271032.

55. Kaneto H, Fujii J, Seo HG, Suzuki K, Matsuoka T, et al. (1995) Apoptotic cell death triggered by nitric oxide in pancreatic beta-cells. Diabetes 44: 733-738.

56. Johnson AS, O'Sullivan E, D'Aoust LN, Omer A, Bonner-Weir S, et al. (2011) Quantitative assessment of islets of Langerhans encapsulated in alginate. Tissue Eng Part C Methods 17: 435-449.

57. Park KS, Kim YS, Kim JH, Choi B, Kim SH, et al. (2010) Trophic molecules derived from human mesenchymal stem cells enhance survival, function, and angiogenesis of isolated islets after transplantation. Transplantation 89: 509517

58. Vériter S, Gianello P, Igarashi Y, Beaurin G, Ghyselinck A, et al. (2014) Improvement of subcutaneous bioartificial pancreas vascularization and function by coencapsulation of pig islets and mesenchymal stem cells in primates. Cell Transplant 23: 1349-1364.

59. Lu Y1, Jin X, Chen Y, Li S, Yuan Y, et al. (2010) Mesenchymal stem cells protect islets from hypoxia/reoxygenation-induced injury. Cell Biochem Funct 28: 637-
60. Vériter S, Aouassar N, Adnet PY, Paridaens MS, Stuckman C (2011) The impact of hyperglycemia and the presence of encapsulated islets on oxygenation within a bioartificial pancreas in the presence of mesenchymal stem cells in a diabetic Wistar rat model. Biomaterials 32: 5945-5956.

61. Barkai U, Weir GC, Colton CK, Ludwig B, Bornstein SR, et al. (2013) Enhanced oxygen supply improves islet viability in a new bioartificial pancreas. Cel Transplant 22: 1463-1476.

62. Ludwig B, Ziegler CG, Schally AV, Richter C, Steffen A, et al. (2010) Agonis of growth hormone-releasing hormone as a potential effector for survival and proliferation of pancreatic islets. Proc Natl Acad Sci U S A 107: 12623-12628.

63. Bloch K, Papismedov E, Yavriyants K, Vorobeychik M, Beer S, et al. (2006) Photosynthetic oxygen generator for bioartificial pancreas. Tissue Eng 12: $337-$ 344.

64. Pedraza E, Coronel MM, Fraker CA, Ricordi C, Stabler CL (2012) Preventing hypoxia-induced cell death in beta cells and islets via hydrolytically activated oxygen-generating biomaterials. Proc Natl Acad Sci U S A 109: 4245-4250.

65. Stiegler P, Matzi V, Pierer E, Hauser O, Schaffellner S, et al. (2010) Creation of a prevascularized site for cell transplantation in rats. Xenotransplantation 17: $379-390$.

66. Sakata N, Chan NK, Ostrowski RP, Chrisler J, Hayes P, et al. (2010) Hyperbaric oxygen therapy improves early posttransplant islet function. Pediatr Diabetes 11: $471-478$ 